\title{
Technology Contribution to Improve Autistic Children Life Quality
}

Zhoe Comas-González, Andrés Sánchez-Comas, Emiro De-La-Hoz-Franco, Kåre Synnes, Joaquín F. Sánchez, Carlos Collazos-Morales

\begin{abstract}
: o review published literature on the use of technology and how it has improved autistic children life style. A systematic review of the English literature was performed using the PRISMA guideline. Papers indexed in WOS and Scopus databases were included, adjusted to a timeline between 2016 and 2020 and focused on mobile technology, interventions, improvement of social behavior and communication and autism, aimed to describe the most used mechanism to improve autistic life style. Thirty two (32) papers were included in the review. We obtained 14 papers on the Scopus database and 18 on the WOS database. The majority of studies evidenced the use of virtual reality, mobile devices, video modelling and robots as the most common applications for autism therapies. Technology has caused an improvement in autistic children life quality. The development of mobile applications, virtual reality applications and robots have showed a positive impact reflected in the performance of daily activities and a better understanding of how they feel, how to behave, how to express themselves and interact with others. Technology gives the opportunity to monitor children status; and offers adaptability, safety, and accuracy of the information.
\end{abstract}

Keywords: Autism spectrum disorder, Autism therapies, Technology intervention, Review of the literature 